\title{
Neuroplasticity and depression: Rewiring the brain's networks through pharmacological therapy (Review)
}

\author{
IOANA RĂDULESCU ${ }^{1}$, ANA MIRUNA DRĂGOI ${ }^{2 *}$, SIMONA CORINA TRIFU $^{3 *}$ and MIHAI BOGDAN CRISTEA ${ }^{4 *}$ \\ ${ }^{1}$ Faculty of Psychology and Educational Sciences, University of Bucharest, 050663 Bucharest; ${ }^{2}$ Department of Psychiatry, \\ 'Prof. Dr. Alex. Obregia' Clinical Hospital of Psychiatry, 041914 Bucharest; Departments of ${ }^{3}$ Clinical Neurosciences, \\ ${ }^{4}$ Morphological Sciences, 'Carol Davila' University of Medicine and Pharmacy, 020021 Bucharest, Romania
}

Received June 18, 2021; Accepted July 19, 2021

DOI: $10.3892 /$ etm.2021.10565

\begin{abstract}
In modern society, depression is one of the most common mental illness; however, its pathophysiology is not yet fully understood. A great body of evidence suggests that depression causes changes in neuroplasticity in specific regions of the brain which are correlated to symptom severity, negative emotional rumination as well as fear learning. Depression is correlated with atrophy of neurons in the cortical and limbic brain regions that control mood and emotion. Antidepressant therapy can exhibit effects on neuroplasticity and reverse the neuroanatomical changes found in depressed patients. The investigation of fast-acting agents that reverse behavioral and neuronal deficiencies of chronic depression, especially the glutamate receptor antagonist NMDA ketamine, and the cellular mechanisms underlying the rapid antidepressant actions of ketamine and related agents are of real interest in current research. Actual medication such as serotonin (5-HT) selective reuptake inhibitor (SSRI) antidepressants, require weeks to months of administration before a clear therapeutic response. The current review aimed to underline the negative effects of depression on neuroplasticity and present the current findings on the effects of antidepressant medication.
\end{abstract}

\section{Contents}

1. Introduction

2. Research methods

3. The pathological aftermath of depression

4. Neuroplasticity and depression

Correspondence to: Dr Simona Corina Trifu, Department of Clinical Neurosciences, 'Carol Davila' University of Medicine and Pharmacy, 37 Dionie Lupu Street, 020021 Bucharest, Romania E-mail: simona.trifu@umfcd.ro

\section{*Contributed equally}

Key words: depression, psychopharmacology, neuroplasticity, prefrontal cortex, cortisol
5. Neurotransmitters involved in depression

6. The importance of hypothalamic-pituitary dysfunction in depression

7. Rewiring the brain's circuits through antidepressant therapy

8. Conclusions

\section{Introduction}

The World Health Organization estimates that there are approximately 264 million people worldwide that suffer from depression. It is a major public health concern given the fact that 800,000 people die from suicide every year (1). Currently, due to the development of pharmacological therapies, psychiatrists have the means to 'rewire' the brain's circuits in order to improve the patient's quality of life. New studies have discovered the modulatory effect on neuroplasticity of certain antidepressants that target areas in the brain responsible for negative rumination. The following review aims to analyze the existing data and outline future research directions in this field. We start by observing the morphological aspects that have been described in people with depression and outline the stress-induced neuroplasticity-related mechanisms that lead to the anatomical findings. We then discuss the morphological evidence of the effects on neuroplasticity that antidepressants determine.

\section{Research methods}

In this article, we performed a qualitative review of original papers and meta-analyses published during the last 10 years. We searched especially scientific material written in English language, but also those written in the Romanian language as well. The initial review protocol was conducted through Google Scholar search. Having in mind the diversity of the articles, we also searched two major databases PubMed ${ }^{\circledR} /$ MEDLINE, and Web of Science Core Collection. As information sources, we searched the databases PubMed ${ }^{\circledR} /$ MEDLINE (http://www. ncbi.nlm.nih.gov/pubmed) and Web of Science for depression, treatment, and related keywords. We conducted a search in the two databases (filters applied: clinical trial, randomized controlled trial, clinical case series, on humans, in the last 
ten years) with the keywords: 'depression' AND 'neuroplasticity' OR 'cortical disfunction' OR 'neurotransmitters' OR 'serotonin receptors' OR 'treatment-resistant depression' OR 'antidepressant' OR 'HPA' OR 'dopaminergic pathway' OR 'ECT' OR 'Romania'. We restricted our search for articles written in English or Romanian; the last updated search was completed on April 10, 2020.

The 'depression' keyword search retrieved over 66,100 results, 3,872 clinical trials, 1,274 meta-analyses, 2,545 systematic reviews and 3,265 randomized clinical trials showing in this way a persistent interest in this psychiatric field. Narrowing the search to 'depression' AND 'neuroplasticity' there were 1,275 retrieved results. In the same way 'depression' AND 'cortical disfunction' brought 1,361 results. Combining the search with 'treatment resistant depression' the results narrowed to 50 articles. Also interesting was that the combination 'depression' and Romania gave us only 235 articles, 6 meta-analyses and 11 clinical trials, all of them involving several countries, not only Romania. We filtered the search excluding duplicates.

In the same way we searched the Web of Science (WOS) 'depression' keyword search retrieved over 175,745 results, on which 42,394 studies were centered on psychiatry, 17,994 on clinical neurology and 21,992 on neurosciences. Studies about neuroplasticity associated with depression were limited to 663 results. 'Treatment resistant depression' provided only one result over the WOS. We conducted this in the same way with all the above-mentioned keywords.

Finally, we systematized all these findings and we selected what we considered the relevant information in this present article.

\section{The pathological aftermath of depression}

Currently, psychiatrists diagnose depression by observing the patient's clinical manifestations, negative emotions and their low functionality in everyday life. Research has concluded that there are significant alterations in the structure of gray and white matter in the frontal lobe, hippocampus, temporal lobe, thalamus, striatum, and amygdala of depressed individuals. However, the scientific consensus regarding the pathophysiology of depression states that there may be a disruption in the functional circuits connecting different brain regions, rather than one focal anomaly (2).

Meta-analyses have confirmed that the hippocampus of depressive patients is smaller in size than that of healthy individuals (3-6). Since the hippocampus is associated with memory and complex cognitive processes, decreased grey matter in this area could be associated with the negative emotions and impaired cognition in depressed patients.

Prefrontal lesions and thinning have been commonly associated with depression. Brodmann area 24 (a part of the anterior cingulate cortex), orbitofrontal cortex, middle prefrontal cortex, dorsolateral prefrontal cortex and other areas have been studied and have been found to be correlated with symptoms of depression (7-10). These regions are crucial in mood regulation, motivational behavior, executive functions, attention and decision-making. Studies have confirmed that after treatment with antidepressants there is an increase in the volume of grey matter and neural activity in these regions $(11,12)$.
A number of studies have concluded that patients suffering from depression have a decrease in the volume of the thalamus, being responsible for integration of sensorial stimuli, emotions and arousal $(13,14)$.

Studies on patients with major depression found a decrease in the grey matter intensity in the striatum (15). Moreover, the disruption of striatal circuits may lead to suicidal behavior (16). The extrapyramidal system codes reward-orientated behavior, cognitive processes, motivation and emotional control. Decreased levels of grey matter throughout this system may explain the core symptoms of depression $(17,18)$.

In contrast to the findings above mentioned, an increase in cortical thickness in the parietal lobe has been observed $(19,20)$. This region is part of the default mode network (DMN); the exaggerated activation of this circuit has been proven to be at the core of internal self-focus, rumination and high-analyzability of negative emotions in depressive patients (21). Accompanying the increased thickness of the parietal cortex, an increase in the volume of the amygdala has been noted $(22,23)$, along with a hyperactivity in this region $(24,25)$. The hyperactivity of the amygdala was found to be positively correlated with the intensity of negative emotions and with fear learning $(26,27)$.

\section{Neuroplasticity and depression}

Since Michael Merzenich observed that the brain is highly plastic and can change in response to intrinsic and extrinsic factors, the scientific world started developing strategies for maximizing this property (28). When scientists discovered that the human hippocampus is capable of developing new neurons by dividing progenitor cells in the dentate gyrus, the paradigm shifted and hope for new therapeutic methods arose (29).

The general term 'neuroplasticity' refers to a series of processes that can occur in the central nervous system as a response to certain stimuli. It can be described as the brain's capacity to reshape its structure and rewire its connections by strengthening or weakening the synaptic transmission. In addition to this, scientists have observed synapse formation and retraction, spinogenesis, synaptogenesis, axonal sprouting and regeneration, and dendrite growth and formation (30).

However, the plastic property of the brain can also be perceived in a negative manner. In mental illnesses, the synaptic reorganization process can be maladaptive and may lead to the persistence of psychiatric symptoms.

High levels of plasmatic cortisol have been observed to suppress neuroplasticity in the hippocampus (31). Glucocorticoids are known to decrease the apical dendritic branches of pyramidal cells in the hippocampus, exhibiting neurotoxic effects and an inhibiting effect on adult neurogenesis $(32,33)$. A study observed that excessive glucocorticoids can impair glial cell functions by inhibiting glucose transport in astrocytes, thus limiting their capacity to aid neurons and to clear the synapses of possibly damaging glutamate accumulation (34).

Similar to the hippocampal effect, elevated levels of glucocorticoids have been linked to decreased apical dendrites in the medial prefrontal cortex $(35,36)$. Chronic stress can induce debranching of the dendritic arbor of pyramidal cells in the medial prefrontal cortex, and also cause loss of spine density 
in those neurons (37). On account of the inhibitory effect of the prefrontal cortex on the amygdala (38), these changes are associated with impaired fear extinction and deregulation of acquired fear $(39,40)$.

Sadness and negative affect in depressed patients are a result of the disruption of top-down processing; activity in the prefrontal and parietal cortex is decreased and activity in the subgenual cingulate and in the anterior insula is increased (41). Dysregulation of these neurological pathways can be the reason for altered emotion responses to negative stimuli. Consequently, a study revealed that emotional processing in depression is diverted to the dorsal insula, a region that is responsible for pain processing in healthy people, this finding being a possible explanation for emotional allodynia in depressed patients (42).

While stress and high levels of cortisol can induce atrophy in some regions in the brain, research has found that prolonged stress can induce the development of dendritic spines and synaptic connectivity in the amygdala (25). This particular negative neuroplasticity effect is known to enhance the rumination of negative emotion and fear-learning mechanisms.

More recently, studies conducted on the DMN showed great variability of this system in the pathology of depression. The DMN is an interconnection of different brain circuits that is activated during restful states, self-referral thinking and also when foreseeing emotional response to certain situations, and planning future activities by taking into consideration other person's beliefs and intentions $(43,44)$. Recent studies have observed that depressed patients present overactivation of the DMN, thus contributing to rumination of negative emotions (45).

An interesting study tried to pinpoint the dysfunctionality of different brain networks in association to a variety of symptoms of depression. It demonstrated the existence of 8 neural biotypes that are responsible for rumination, anxious avoidance, negative bias, threat dysregulation, anhedonia, context insensitivity, inattention and cognitive dyscontrol. The analysis of the neural circuits underlining each biotype can be a clinical guide in order to maximize and predict the response to treatment. As an example, anhedonia is mediated through hypoactivation of the striatal pathways and depressed patients that exhibit this symptom may benefit from medication targeting neuroplasticity in the striatal dopaminergic circuits (such as pramipexole) (46-48).

The small interhemispheric bundles are placed on two planes, one lower and one higher. The lower plane is located at the level of the third ventricle, here is Gudden's commissure, which connects the two internal geniculate bodies, Meynert's commissure, which unites the two sublenticular regions, and the subthalamic commissure, which unites the two subthalamic regions. In the upper plane are the posterior white commissure at the level of the epiphysis, which is a mesencephalic commissure, the inter-habenular commissure, which unites the two ganglia of the habenula, and the anterior white commissure, which unites the temporal lobes (46).

The large interhemispheric bundles are represented by the cerebral trigone (fornix) and the corpus callosum. The cerebral trigone (fornix) consists of fibers that connect the areas of the archicortex, being placed on the third ventricle and the thalamus. The corpus callosum (corpus calosum) is the most important interhemispheric commissure. The corpus callosum has an important role in psychic, motoric and gnostic functions, which require the participation of the two cerebral hemispheres. Experimental sectioning of the corpus callosum was found to lead to the loss of the possibility of inter-hemispheric transfer of information (46).

The interhemispheric circuits that connect the right hemisphere to the left hemisphere work with the white matter fibers of the corpus callosum, and studies using nuclear magnetic resonance show that their absence from birth, craniocerebral trauma and some mental disorders are associated with a significantly low white matter in the area of the corpus callosum (49). In addition, chronic stress and depression are able to cause significant changes in the brain, such as decreased number of dendrites and synapses, atrophy of dendrites and decrease in both size and number of glial cells in the hippocampus and prefrontal cortex (50). Therefore, in this context in which changes can occur at the cognitive and behavioral level, due to the organic changes produced in the brain, the neuroplasticity capacity of the brain is taken into account.

Certain antidepressants help the mechanisms of neuroplasticity and work by repairing damage to neural circuits, strengthening synapses in certain brain areas or normalizing glutamatergic levels. Moreover, the organic changes at the synapses can be based on the fibers covered with muscles and the pyramidal neurons of the hippocampus (51). Therefore, in the case of major depressive disorder, the neuroplasticity capacity of the brain is affected, and this involves difficulties in the hippocampus, involved in learning and memory, the prefrontal cortex involved in attention, concentration, learning and memory and in the amygdala, which plays a role in emotion processing and social learning (52). Regarding the intrahemispheric circuits, the neuroplasticity capacity of the brain is supported by the hippocampus, amygdala and prefrontal cortex. Regarding the hippocampal area, the withdrawal of dendrites and synapses causes a decrease in neuron-synapse connectivity, which makes possible the continuity of information transmission at the level of neurons. Regarding the prefrontal cortex, patients diagnosed with major depressive disorder demonstrate a significant reduction of the dendritic spine, atrophy of the dendritic branches, a decrease in the number of synapses and glial cells (53). In the case of the amygdala, an increase in the number of dendrites was observed in the basolateral nucleus of the amygdala and in the pyramidal projections of the stellar neurons (54).

\section{Neurotransmitters involved in depression}

Serotonin-serotonergic neurons project to the entire central nervous system. L-tryptophan is converted by tryptophan hydroxylase to serotonin. It has been observed that inhibition of this reaction is associated with depression-like manifestations, probably due to serotonin depletion.

Norepinephrine. Depletion of norepinephrine by inhibiting tyrosine hydroxylase has shown that norepinephrine is involved in depression (55).

Dopamine. It has been observed that patients with Parkinson's disease have depressive episodes, and reserpine (decreases 
monoamine vesicles) can induce depression as an adverse reaction. This has led to the hypothesis that decreased activation of dopaminergic pathways can cause depression in some patients. Monoamine oxidase inhibitors were among the first antidepressants, increasing the availability of dopamine, serotonin and norepinephrine. Complex interactions take place between these neurotransmitters, and alteration of one system of monoamines can affect the others (55).

Glutamate. Some antidepressants that target mainly monoamines may reduce the functionality of glutamatergic $\mathrm{N}$-methyl-D-aspartate (NMDA) and $\alpha$-amino-3-hydroxy-5methyl-4-isoxazolepropionic acid (AMPA) receptors.

Glycine. The glycine site of N-methyl-d-aspartate receptors (NMDARs) can be a target to ameliorate the symptoms of depression and cognitive dysfunction. Six glycine site modulators with pro-cognitive and antidepressant properties have been identified: d-serine (co-agonist), d-cycloserine (partial agonist), d-alanine (co-agonist), glycine (agonist), sarcosine (co-agonist) and rapastinel (partial agonist) (56).

Depression and anxiety are associated with deficiency of neurotransmitters (monoamines) and abnormal functioning of their receptors. Disruption of the systems of the three neurotransmitters, serotonin, norepinephrine and dopamine, can have effects on different areas of the brain.

Impairment of serotonin (5-HT) receptor synthesis, release, or transport may exacerbate depression. Previous studies have found that serum 5-HT concentrations in patients with depression were found to be significantly lower than those from normal tests, suggesting 5-HT deficiency in patients with depression (57). Similarly, post-mortem studies have shown low concentrations of 5-HT and its major metabolite 5-HIAA in the brain tissue of depressed and suicidal patients. Seven families of 5-HT receptors have been identified, including 5-HT1 to 5-HT7 receptors with their subtypes. Numerous studies have systematically conceptualized that abnormalities of the 5-HT1 (5-HT1A, 5-HT1D) and 5-HT2 (5-HT2A, 5-HT2C) receptors in the central nervous system may explain the manifestations of depression (58). Several important regions of the brain, such as the prefrontal cortex, amygdala, and hippocampus are involved in the pathophysiology of depression. The insular cortex is also considered crucial in explaining emotional deficits in depression (2). Analyses showed that people with depression showed abnormal activity in the dorsal island while anticipating painful stimuli, as opposed to healthy people (21). Acute stimulation of the 5-HT2A and 5-HT2C receptors projecting from the pontine raphe nucleus to the amygdala and the marginal limbic cortex can cause acute psychomotor agitation, anxiety, and panic attacks (59).

The norepinephrine hypothesis has been proposed in depression; namely that depressive symptoms are caused by a decrease in the central nervous system. Post-mortem studies in patients with depression found an increased conformation of central $\alpha 2$-adrenergic autoreceptors and a low affinity for binding of the norepinephrine transporter to the locus coeruleus, without any significant change in the density of $\alpha 2$-adrenergic receptors in the raffia nuclei (60). Reduction of norepinephrine in the central nervous system is associated with depletion of positive affective resources in patients with depression, including decreased pleasure, interest, energy, passion, and loss of confidence.

Dopamine, a neurotransmitter in the hypothalamus and pituitary gland, is a neurobiological substrate for reward, psychomotor speed, and the ability to experience pleasure. Their lack is associated with depression. Low activity of dopamine-releasing neurons can induce symptoms of depression. Decreased activity of dopamine receptor function can lead to inability to inhibit from the prefrontal cortex to the amygdala, resulting in overexcitation of the amygdala, which causes fear and anxiety. Dopamine receptors are type D1 and D2. Studies have shown a low density of dopamine transporters and an inefficient binding of D2 receptors in striated bodies in anxious patients. Moreover, the level of homovanillic acid, a metabolite of dopamine in the cerebrospinal fluid, was found to have a lower level in patients with depression (61).

In the case of major depressive disorder, but also milder cases of depression, it appears that dopamine, norepinephrine and serotonin are involved in both maintaining the mechanisms of depression and depressive symptoms (55). Specific manifestations are associated with an increase or decrease in certain neurotransmitters, which suggests that certain symptoms are associated with specific neurochemical mechanisms. A two-dimensional model of neurotransmitter functions in depression describes this disorder as a combination of increasing negative affect and decreasing positive affect (62), which correlates with sadness and mood swings.

Dopamine creates positive feelings, associated with reward, which motivates individuals in work tasks. At the neuronal level, the synthesis and release of dopamine are achieved with the help of dopamine neurons through dopaminergic pathways, which are involved in executive function, learning, reward motivation and neuroendocrine control (63).

The mesolimbic pathway transmits dopamine from the ventral tegmental area to the ventral striatum and is involved in the response to pleasure, positive reinforcement, aversion processing, and a sense of gratitude for the reward. The mesocortical pathway transmits dopamine from the entered tegmental area to the prefrontal cortex and is involved in executive functions. The nigrostriatal pathway transmits dopamine from the substantia nigra, from the midbrain, to the caudate nucleus and putamen and are involved in associative learning and motor function. The tuberoinfundibular pathway transmits dopamine from the arcuate nucleus of the hypothalamus to the pituitary gland, which inhibits the release of prolactin from the pituitary gland. The hypothalamospinal tract influences the locomotor network in the brainstem and spinal cord, implication in motor function. The uncertain hypothalamic pathway influences visceral and sensorimotor activity (64).

In the case of major depressive disorder, several studies support a low neurotransmission of dopamine in the brain. At the physiological level, the low dopamine transmission is due either to the fact that presynaptic neurons release a low dose of dopamine, or to the impairment of the functioning of receptors in the synaptic cleft. Norepinephrine or noradrenaline is synthesized by dopamine and is secreted by the adrenal glands and adrenergic nerve pathways. Noradrenaline is secreted as a result of stimuli that the person perceives as stressful and that act on the body in several ways. It increases the heart rate, redirects blood flow to the muscles, increases blood glucose 
levels and ensures a higher energy intake. In the case of major depressive disorder, but also milder cases of depression, a low level of norepinephrine has been observed, associated with difficulties in mobilizing for activity, attention or energy (65).

Serotonin is associated with mood and mood swings. In the case of depressive disorders, it has been observed that serotonin levels are generally lower than in people without depressive symptoms (66). This hypothesis is based on the concept of tryptophan depletion, which causes mood swings and is associated with depression (67).

\section{The importance of hypothalamic-pituitary dysfunction in depression}

The stress response is necessary to maintain homeostasis, but long-term activation of the hypothalamic-pituitary-adrenal (HPA) system increases the risk of depression. The neural network that evaluates stressful events consists of the HPA, the arginine-vasopressin system and the noradrenergic system. It is hyperactive through a combination of genetic factors, developing sequelae and events that generate negative emotions. Thus, major depression occurs through the interaction of vulnerable genes and environmental factors. Prenatal stressors, such as placental insufficiency or nicotine exposure, may increase the risk of depression. Psychological stress, such as premature separation of the child from the mother or abuse can lead to an increase in the activity of the HPA, increasing the risk of depression. When the HPA is activated, cortisol synthesis increases. An increased proportion of depressed patients has been observed in cases of Cushing's disease or during treatment with high doses of synthetic corticosteroids (68).

In the event of a dysfunction in the hypothalamic-pituitary system, this can lead to certain conditions in which depressive symptoms develop. For example, pituitary insufficiency syndrome occurs when pituitary hormones are not secreted in the required amount. The hormonal secretion of pituitary hormones is regulated by the hypothalamus, by stimulating and inhibiting hormones and by hormones secreted by stimulation of the glands in the periphery. Pituitary pathology, in general, defines inhibitory states, depression, neurotic states, in fact depressive disorders. The HPA axis involves a constant feedback loop among the hypothalamus, pituitary and adrenal glands. During a physically or emotionally stressful event, the HPA axis is activated, and the hypothalamus secretes two hormones, corticotropin and vasopressin, which act on the pituitary gland to secrete adrenocorticotropin hormone (ACTH). ACTH is transported through the blood vessels to the adrenal cortex and interacts with adrenocortical cell receptors, which stimulate the production and release of cortisol. Although the HPA axis is the basis of the stress response to danger, a mechanism involved in adaptation and evolution, prolonged and excessive stress can cause disorders or induce vulnerabilities. In depressive diseases, an excess of cortisol in the blood has been observed, an increased level of corticotropin and adrenocorticotropin (69). A recent meta-analysis suggests that depressive pathologies differ in terms of HPA axis reactivity, where depressed patients with melancholic features tend to have axis hyperactivation and those with atypical depression tend to have low axis activity (70).

\section{Rewiring the brain's circuits through antidepressant therapy}

An important discovery in patients who have undergone antidepressant therapy is an increase in the functional connectivity in both neocortical regions (different parts of the prefrontal cortex) and subcortical regions (hippocampus, parahippocampus, insula, orbitofrontal cortex, anterior cingulate cortex); furthermore, this was correlated with clinical improvement of depression symptoms.

Other findings in these patients include decreased activation in specific limbic areas in response to negative stimuli, increased activity in the dorsolateral prefrontal cortex and visual-processing areas in response to positive stimuli. These indicate that antidepressant therapy reverses the hyperactivity of the DMN and the hypoactivity of neocortical regions observed in depressed patients.

In addition, it was demonstrated that after antidepressant therapy there was a decrease in activation of the amygdala during emotional processing, suggesting that it may reduce the negative emotional processing (71). Furthermore, it seems to normalize the activity of regions involved in the resting state and emotional processing and response.

Studies have demonstrated the reverse neuroplasticity effects of antidepressant therapies. Antidepressants were observed to promote neurogenesis in the hippocampus and to strengthen functional connectivity $(72,73)$.

A comparative study observed that patients treated with selective serotonin reuptake inhibitors (citalopram, escitalopram) showed decreased limbic activity and normalization of the frontoparietal network activity during a cognitive task. The same study concluded that serotonin-norepinephrine reuptake inhibitors showed limited impact on the same limbic regions (74).

The biomolecular mechanisms of neuroplasticity that are related to depression and antidepressant therapy are still unclear. Antidepressants may promote neuroplasticity by altering the cellular signaling. This effect is mediated by an increase in levels of neurotrophic factors, such as brain-derived neurotrophic factor (BDNF), which activates the tyrosine kinase receptors and triggers an intracellular cascade involving cAMP-dependent protein kinase A, mitogen-activated protein kinase and other chemicals. These molecules activate transcription factors such as cAMP-responsive element binding-protein and promote synthesis of proteins. The end results are structural changes in specific regions in the brain $(75,76)$.

It was noted that synaptic serotonin availability has a key role in the stress-induced dendritic remodeling (77). Increased synaptic serotonin can trigger intracellular mechanisms that enhance neural plasticity. Treatment with escitalopram was proved to increase the serum level of brain-derived neurotrophic factor (BDNF) (78), therefore promoting neurogenesis in the hippocampus (79). BDNF's mechanism of action is controversial and it was observed that its neuroplasticity effects are region-specific and interact with other genetic and environmental factors (80).

It was observed that glutamate mediates reversible neural modifications (33). Inhibition of glutamate release prevents remodeling of neurons in specific regions in the brain $(81,82)$. By interacting with the NMDA receptor, treatment with 
tianeptine was demonstrated to prevent the retraction of apical dendrites of hippocampal pyramidal neurons and to increase granule cell proliferation (83). Treatment with fluoxetine showed similar results in blocking the effects of stress in the prefrontal cortex (84).

A new antidepressant, ketamine, an NMDA glutamate receptor antagonist, has gained attention in recent years as it is regarded as a possible new antidepressant treatment. A single subanesthetic dose was observed to have a $52.6 \%$ response rate and lasts up to 3 days (85). In an unpublished document by Clarke and Hayley, it was stipulated that ketamine alters the level of serotonin in the prefrontal cortex during basal state (86). This response to ketamine is dependent upon an intact serotoninergic system (87). It was confirmed that chronic ketamine intake upregulates dopamine synthesis and levels (88). Ketamine has also elicited effects on the structure and function of the prefrontal cortex synapses. It was demonstrated that it can enhance dendritic branching and synaptic receptor number within $24 \mathrm{~h}$ of ketamine administration (89). An important study observed the beneficial and synergistic antidepressant effects of combining ketamine and electroconvulsive therapy (90). The primary concern regarding ketamine therapy is the psychotomimetic effects.

Electroconvulsive therapy (ECT) is considered the last resort in medication-refractory depression. Studies have confirmed that ECT induces neuroplasticity through different mechanism such as: synaptogenesis, neurogenesis, dendrogenesis, angiogenesis and gliogenesis (91). These changes occur in areas connected to the prefrontal cortex and the limbic system that are involved in mood regulation. The volumes of the hippocampus and amygdala were found to be normalized after one course of ECT. Apart from the structural changes that were observed, ECT is associated with 'rewiring' of the brain in a functional manner. A study demonstrated the effect of ECT in normalizing resting state functional connectivity within the brain's networks, that was noted as early as the first session of ECT (92). This suggests that, initially, there is a decrease in intra-limbic networks following ECT. Subsequently, changes extend to increase the functional connectivity between the prefrontal cortex and limbic system restoring the top-down regulation circuits. A reduction in the DMN hyperactivity, correlated with improvement in the symptoms, was also observed (92).

\section{Conclusions}

Antidepressant therapy has the power to overdrive the negative neuroplasticity caused by depression. All patients suffering from depression should benefit from antidepressant therapy in order to achieve clinical remission. Recent studies have shown that clinical remitted patients who have benefited from antidepressant therapy have a better quality of life. Special attention must be drawn to non-pharmacological methods that can enhance the antidepressant effects and provide psychological means to overcome other stressful situations in life.

\section{Acknowledgements}

Not applicable.

\section{Funding}

No funding was received.

\section{Availability of data and materials}

Not applicable.

\section{Authors' contributions}

SCT and IR designed and drafted the initial review. IR and AMD gathered the medical information and conducted the final view and structure of the article and investigated the present area of research and gathered the important information. SCT and MBC finalized the work, conducted analysis of the results and approved the final version of the work. All authors read and approved the final manuscript for publication.

\section{Ethics approval and consent to participate}

Not applicable.

\section{Patient consent for publication}

Not applicable.

\section{Competing interests}

The authors declare that they have no competing interests.

\section{References}

1. World Health Organization: Information on Mental Disorders. Available from: http://www.who.int/mental_health/management/ depression/definition/en/index.

2. Zhang FF, Peng W, Sweeney JA, Jia ZY and Gong QY: Brain structure alterations in depression: Psychoradiological evidence. CNS Neurosci Ther 24: 994-1003, 2018.

3. Geerlings MI and Gerritsen L: Late-life depression, hippocampal volumes, and hypothalamic-pituitary-adrenal axis regulation: A systematic review and meta-analysis. Biol Psychiatry 82: 339-350, 2017.

4. Campbell S, Marriott M, Nahmias C and MacQueen GM: Lower hippocampal volume in patients suffering from depression: A meta-analysis. Am J Psychiatry 161: 598-607, 2004.

5. Eker C and Gonul AS: Volumetric MRI studies of the hippocampus in major depressive disorder: Meanings of inconsistency and directions for future research. World J Biol Psychiatry 11: 19-35, 2010.

6. Trifu S: Neuroendocrine insights into burnout syndrome. Acta Endocrinol (Bucur) 15: 404-405, 2019.

7. Srivastava S, Bhatia MS, Bhargava SK, Kumari R and Chandra S: A diffusion tensor imaging study using a voxel-based analysis, region-of-interest method to analyze white matter abnormalities in first-episode, treatment-naïve major depressive disorder. J Neuropsychiatry Clin Neurosci 28: 131-137, 2016.

8. Bora E, Fornito A, Pantelis C and Yücel M: Gray matter abnormalities in major depressive disorder: A meta-analysis of voxel based morphometry studies. J Affect Disord 138: 9-18, 2012.

9. Ramezani M, Abolmaesumi P, Tahmasebi A, Bosma R, Tong R, Hollenstein T, Harkness K and Johnsrude I: Fusion analysis of first episode depression: Where brain shape deformations meet local composition of tissue. Neuroimage Clin 7: 114-121, 2015.

10. Foland-Ross LC, Sacchet MD, Prasad G, Gilbert B, Thompson PM and Gotlib IH: Cortical thickness predicts the first onset of major depression in adolescence. Int J Dev Neurosci 46: 125-131, 2015.

11. Shen Z, Cheng Y, Yang S, Dai N, Ye J, Liu X, Lu J, Li N, Liu F, $\mathrm{Lu} \mathrm{Y}$, et al: Changes of grey matter volume in first-episode drug-naive adult major depressive disorder patients with different age-onset. Neuroimage Clin 12: 492-498, 2016. 
12. Kong L, Wu F, Tang Y, Ren L, Kong D, Liu Y, Xu K and Wang F: Frontal-subcortical volumetric deficits in single episode, medication-naïve depressed patients and the effects of 8 weeks fluoxetine treatment: A VBM-DARTEL study. PLoS One 9: e79055, 2014

13. Lu Y, Liang H, Han D, Mo Y, Li Z, Cheng Y, Xu X, Shen Z, Tan C, Zhao W, et al: The volumetric and shape changes of the putamen and thalamus in first episode, untreated major depressive disorder. Neuroimage Clin 11: 658-666, 2016.

14. Zhang H, Li L, Wu M, Chen Z, Hu X, Chen Y, Zhu H, Jia Z and Gong Q: Brain gray matter alterations in first episodes of depression: A meta-analysis of whole-brain studies. Neurosci Biobehav Rev 60: 43-50, 2016

15. Jacobs RH, Barba A, Gowins JR, Klumpp H, Jenkins LM, Mickey BJ, Ajilore O, Peciña M, Sikora M, Ryan KA, et al: Decoupling of the amygdala to other salience network regions in adolescent-onset recurrent major depressive disorder. Psychol Med 46: 1055-1067, 2016.

16. Dombrovski AY, Siegle GJ, Szanto K, Clark L, Reynolds CF and Aizenstein H: The temptation of suicide: Striatal gray matter, discounting of delayed rewards, and suicide attempts in late-life depression. Psychol Med 42: 1203-1215, 2012.

17. Wagner G, Koch K, Schachtzabel C, Schultz CC, Sauer H and Schlösser RG: Structural brain alterations in patients with major depressive disorder and high risk for suicide: Evidence for a distinct neurobiological entity? Neuroimage 54: 1607-1614, 2011.

18. Forbes EE, Hariri AR, Martin SL, Silk JS, Moyles DL, Fisher PM Brown SM, Ryan ND, Birmaher B, Axelson DA and Dahl RE: Altered striatal activation predicting real-world positive affect in adolescent major depressive disorder. Am J Psychiatry 166 : 64-73, 2009.

19. Yang XH, Wang Y, Huang J, Zhu CY, Liu XQ, Cheung EF Xie GR and Chan RC: Increased prefrontal and parietal cortical thickness does not correlate with anhedonia in patients with untreated first-episode major depressive disorders. Psychiatry Res 234: 144-151, 2015

20. Chen Z, Peng W, Sun H, Kuang W, Li W, Jia Z and Gong Q High-field magnetic resonance imaging of structural alterations in first-episode, drug-naive patients with major depressive disorder. Transl Psychiatry 6: e942, 2016.

21. Sliz D and Hayley S: Major depressive disorder and alterations in insular cortical activity: A review of current functional magnetic imaging research. Front Hum Neurosci 6: 323, 2012.

22. Lange $C$ and Irle E: Enlarged amygdala volume and reduced hippocampal volume in young women with major depression. Psychol Med 34: 1059-1064, 2004

23. Frodl T, Meisenzahl E, Zetzsche T, Bottlender R, Born C, Groll C, Jäger M, Leinsinger G, Hahn K and Möller HJ: Enlargement of the amygdala in patients with a first episode of major depression. Biol Psychiatry 51: 708-714, 2002.

24. Drevets WC, Videen TO, Price JL, Preskorn SH, Carmichael ST and Raichle ME: A functional anatomical study of unipolar depression. J Neurosci 12: 3628-3641, 1992.

25. Vyas A, Jadhav S and Chattarji S: Prolonged behavioral stress enhances synaptic connectivity in the basolateral amygdala. Neuroscience 143: 387-393, 2006.

26. Abercrombie HC, Schaefer SM, Larson CL, Oakes TR, Lindgren KA, Holden JE, Perlman SB, Turski PA, Krahn DD, Benca RM and Davidson RJ: Metabolic rate in the right amygdala predicts negative affect in depressed patients. Neuroreport 9 : 3301-3307, 1998 .

27. Conrad CD, LeDoux JE, Magariños AM and McEwen BS: Repeated restraint stress facilitates fear conditioning independently of causing hippocampal CA3 dendritic atrophy. Behav Neurosci 113: 902-913, 1999.

28. Merzenich M: Soft-Wired: How the new science of brain plasticity can change your life. San Francisco:Parnassus Publishing, 2013.

29. Eriksson PS, Perfilieva E, Björk-Eriksson T, Alborn AM, Nordborg C, Peterson DA and Gage FH: Neurogenesis in the adult human hippocampus. Nat Med 4: 1313-1317, 1998.

30. Albert PR: Adult neuroplasticity: A new 'cure' for major depression? J Psychiatry Neurosci 44: 147-150, 2019.

31. Sapolsky RM: Glucocorticoids and hippocampal atrophy in neuropsychiatric disorders. Arch Gen Psychiatry 57: 925-935, 2000.

32. Woolley CS, Gould E and McEwen BS: Exposure to excess glucocorticoids alters dendritic morphology of adult hippocampal pyramidal neurons. Brain Res 531: 225-231, 1990.
33. Duman RS: Depression: A case of neuronal life and death? Biol Psychiatry 56: 140-145, 2004.

34. Virgin CE Jr, Ha TP, Packan DR, Tombaugh GC, Yang SH, Horner HC and Sapolsky RM: Glucocorticoids inhibit glucose transport and glutamate uptake in hippocampal astrocytes: Implications for glucocorticoid neurotoxicity. J Neurochem 57: 1422-1428, 1991.

35. Radley JJ, Sisti HM, Hao J, Rocher AB, McCall T, Hof PR, McEwen BS and Morrison JH: Chronic behavioral stress induces apical dendritic reorganization in pyramidal neurons of the medial prefrontal cortex. Neuroscience 125: 1-6, 2004.

36. Wellman CL: Dendritic reorganization in pyramidal neurons in medial prefrontal cortex after chronic corticosterone administration. J Neurobiol 49: 245-253, 2001.

37. Radley JJ, Rocher AB, Rodriguez A, Ehlenberger DB, Dammann M, McEwen BS, Morrison JH, Wearne SL and Hof PR: Repeated stress alters dendritic spine morphology in the rat medial prefrontal cortex. J Comp Neurol 507: 1141-1150, 2008.

38. Furini C, Myskiw J and Izquierdo I: The learning of fear extinction. Neurosci Biobehav Rev 47: 670-683, 2014.

39. Izquierdo A, Wellman CL and Holmes A: Brief uncontrollable stress causes dendritic retraction in infralimbic cortex and resistance to fear extinction in mice. J Neurosci 26: 5733-5738, 2006

40. Dibbets P, van den Broek A and Evers EA: Fear conditioning and extinction in anxiety- and depression-prone persons. Memory 23 : 350-364, 2015

41. Mayberg HS, Liotti M, Brannan SK, McGinnis S, Mahurin RK, Jerabek PA, Silva JA, Tekell JL, Martin CC, Lancaster JL and Fox PT: Reciprocal limbic-cortical function and negative mood: Converging PET findings in depression and normal sadness. Am J Psychiatry 156: 675-682, 1999.

42. Mutschler I, Ball T, Wankerl J and Strigo IA: Pain and emotion in the insular cortex: Evidence for functional reorganization in major depression. Neurosci Lett 520: 204-209, 2012.

43. Raichle ME, MacLeod AM, Snyder AZ, Powers WJ, Gusnard DA and Shulman GL: A default mode of brain function. Proc Natl Acad Sci USA 98: 676-682, 2001.

44. Jovanov E: On physiological bases of states of expanded in consciousness. In: State of consciousness. Experimental insights into meditation, walking, sleeping and dreams. Cvetkovic D and Cosic I (eds). Springer-Verlag Berlin Heidelberg, New York, NY, pp203-223, 2011.

45. Hamilton JP, Farmer M, Fogelman P and Gotlib IH: Depressive rumination, the default-mode network, and the dark matter of clinical neuroscience. Biol Psychiatry 78: 224-230, 2015.

46. Williams LM: Precision psychiatry: A neural circuit taxonomy for depression and anxiety. Lancet Psychiatry 3: 472-480, 2016.

47. Dragoi AM, Radulescu I, Năsui BA, Pop AL, Varlas VN and Trifu S: Clozapine: An updated overview of pharmacogenetic biomarkers, risks, and safety-particularities in the context of COVID-19. Brain Sci 10: 840, 2020.

48. Pop AL, Crişan S, Bârcă M, Ciobanu AM, Varlas VN, Pop C, Pali MA, Cauni D, Ozon EA, Udeanu D, et al: Evaluation of dissolution profiles of a newly developed solid oral immediate-release formula containing alpha-lipoic acid. Processes 9: 176, 2021.

49. Zarei M, Johansen-Berg H, Smith S, Ciccarelli O, Thompson AJ and Matthews PM: Functional anatomy of interhemispheric cortical connections in the human brain. J Anat 209: 311-320, 2006.

50. Serafini G: Neuroplasticity and major depression, the role of modern antidepressant drugs. World J Psychiatry 2: 49-57, 2012.

51. Popov VI, Bocharova LS and Bragin AG: Repeated changes of dendritic morphology in the hippocampus of ground squirrels in the course of hibernation. Neuroscience 48: 45-51, 1992

52. McEwen BS, Nasca C and Gray JD: Stress effects on neuronal structure: Hippocampus, amygdala, and prefrontal cortex. Neuropsychopharmacology 41: 3-23, 2016.

53. Fuchs E: Neuroplasticity: A new approach to the pathophysiology of depression. In: Neuroplasticity: New biochemical mechanisms. Costa e Silva JA, Macher JP and Olie JP (eds). Springer, London, pp1-12, 2009.

54. Jay TM: Cellular plasticity and the pathophysiology of depression. In: Neuroplasticity: New Biochemical Mechanisms. Costa e Silva JA, Macher JP and Olie JP (eds). Springer, London, pp41-55, 2009.

55. Waløen K, Kleppe R, Martinezand A and Haavik J: Tyrosine and tryptophan hydroxylases as therapeutic targets in human disease. Expert Opin Ther Targets 21: 167-180, 2017. 
56. Peyrovian B, Rosenblat JD, Pan Z, Iacobucci M, Brietzke E and McIntyre RS: The glycine site of NMDA receptors: A target for cognitive enhancement in psychiatric disorders. Prog Neuropsychopharmacol Biol Psychiatry 92: 387-404, 2019.

57. Saldanha D, Kumar N, Ryali V, Srivastava K and Pawar AA: Serum serotonin abnormality in depression. Med J Armed Forces India 65: 108-112, 2009.

58. Villas-Boas GR, Lavorato SN, Paes MM, de Carvalho PMG, Rescia VC, Cunha MS, de Magalhães-Filho MF, Ponsoni LF, de Carvalho AAV, de Lacerda RB, et al: Modulation of the serotonergic receptosome in the treatment of anxiety and depression: A narrative review of the experimental evidence. Pharmaceuticals (Basel) 14: 148, 2021.

59. Tang L, Wang Y, Chen Y, Chen L, Zheng S, Bao M, Xiang J, Luo H, $\mathrm{Li} \mathrm{J}$ and $\mathrm{Li}$ Y: The association between 5HT2A T102C and behavioral and psychological symptoms of dementia in Alzheimer's disease: A meta-analysis. Biomed Res Int 2017: 5320135, 2017.

60. Liu Y, Zhao J and Guo W: Emotional roles of mono-aminergic neurotransmitters in major depressive disorder and anxiety disorders. Front Psychol 9: 2201, 2018.

61. Cervenka S, Hedman E, Ikoma Y, Djurfeldt DR, Rück C, Halldin C and Lindefors N: Changes in dopamine D2-receptor binding are associated to symptom reduction after psychotherapy in social anxiety disorder. Transl Psychiatry 2: e120, 2012.

62. Pöldinger W, Calanchini B and Schwarz W: A functional-dimensional approach to depression: Serotonin deficiency as a target syndrome in a comparison of 5-hydroxytryptophan and fluvoxamine. Psychopathology 24: 53-81, 1991.

63. Bromberg-Martin ES, Matsumoto M and Hikosaka O: Dopamine in motivational control: Rewarding, aversive, and alerting. Neuron 68: 815-834, 2010.

64. Olguín HJ, Guzmán DC, García EH and Mejía GB: The role of dopamine and its dysfunction as a consequence of oxidative stress. Oxid Med Cell Longev 2016: 9730467, 2016.

65. Moret $\mathrm{C}$ and Briley $\mathrm{M}$ : The importance of norepinephrine in depression. Neuropsychiatr Dis Treat 7 (Suppl 1): S9-S13, 2011.

66. Coppen A: The biochemistry of affective disorders. Br J Psychiatry 113: 1237-1264, 1967.

67. Cowen PJ and Browning M: What has serotonin to do with depression? World Psychiatry 14: 158-160, 2015.

68. Pivonello R, De Leo M, Cozzolino A and Colao A: The treatment of cushing's disease. Endocr Rev 36: 385-486, 2015.

69. Nandam LS, Brazel M, Zhou M and Jhaveri DJ: Cortisol and major depressive disorder-translating findings from humans to animal models and back. Front Psychiatry 10: 974, 2020.

70. Juruena MF, Agustini B, Cleare AJ and Young AH: A translational approach to clinical practice via stress-responsive glucocorticoid receptor signaling. Stem Cell Investig 4: 13, 2017.

71. Delaveau P, Jabourian M, Lemogne C, Guionnet S, Bergouignan L and Fossati P: Brain effects of antidepressants in major depression: A meta-analysis of emotional processing studies. J Affect Disord 130: 66-74, 2011.

72. Yan HC, Cao X, Gao TM and Zhu XH: Promoting adult hippocampal neurogenesis: A novel strategy for antidepressant drug screening. Curr Med Chem 18: 4359-4367, 2011

73. Dranovsky A and Hen R: Hippocampal neurogenesis: Regulation by stress and antidepressants. Biol Psychiatry 59: 1136-1143, 2006.

74. Wagner G, Koch K, Schachtzabel C, Sobanski T, Reichenbach JR, Sauer H and Schlösser RG: Differential effects of serotonergic and noradrenergic antidepressants on brain activity during a cognitive control task and neurofunctional prediction of treatment outcome in patients with depression. J Psychiatry Neurosci 35: 247-257, 2010.

75. Andrade $\mathrm{C}$ and Rao NS: How antidepressant drugs act: A primer on neuroplasticity as the eventual mediator of antidepressant efficacy. Indian J Psychiatry 52: 378-386, 2010.
76. Trifu S, Carp EG and Nadoleanu A: Alcohol as a substitute, mask of depression and 'antidote' of narcissism. Eur Proc Soc Behav Sci 31: 986-994, 2017

77. Magariños AM, Deslandes A and McEwen BS: Effects of antidepressants and benzodiazepine treatments on the dendritic structure of CA3 pyramidal neurons after chronic stress. Eur J Pharmacol 371: 113-122, 1999.

78. Cattaneo A, Bocchio-Chiavetto L, Zanardini R, Milanesi E, Placentino A and Gennarelli M: Reduced peripheral brain-derived neurotrophic factor mRNA levels are normalized by antidepressant treatment. Int J Neuropsychopharmacol 13: 103-108, 2010.

79. Santarelli L, Saxe M, Gross C, Surget A, Battaglia F, Dulawa S, Weisstaub N, Lee J, Duman R, Arancio O, et al: Requirement of hippocampal neurogenesis for the behavioral effects of antidepressants. Science 301: 805-809, 2003.

80. Nestler EJ and Carlezon WA Jr: The mesolimbic dopamine reward circuit in depression. Biol Psychiatry 59: 1151-1159, 2006.

81. Watanabe Y, Gould E, Daniels DC, Cameron H and McEwen BS: Tianeptine attenuates stress-induced morphological changes in the hippocampus. Eur J Pharmacol 222: 157-162, 1992.

82. Bonanno G, Giambelli R, Raiteri L, Tiraboschi E, Zappettini S, Musazzi L, Raiteri M, Racagni G and Popoli M: Chronic antidepressants reduce depolarization-evoked glutamate release and protein interactions favoring formation of SNARE complex in hippocampus. J Neurosci 25: 3270-3279, 2005.

83. Malberg JE, Eisch AJ, Nestler EJ and Duman RS: Chronic antidepressant treatment increases neurogenesis in adult rat hippocampus. J Neurosci 20: 9104-9110, 2000.

84. Rocher C, Spedding M, Munoz C and Jay TM: Acute stress-induced changes in hippocampal/prefrontal circuits in rats: Effects of antidepressants. Cereb Cortex 14: 224-229, 2004.

85. Newport DJ, Carpenter LL, McDonald WM, Potash JB, Tohen M and Nemeroff CB; APA Council of Research Task Force on Novel Biomarkers and Treatments: Ketamine and other NMDA antagonists: Early clinical trials and possible mechanisms in depression. Am J Psychiatry 172: 950-966, 2015.

86. Hayley S and Litteljohn D: Neuroplasticity and the next wave of antidepressant strategies. Front Cell Neurosci 7: 218, 2013.

87. Gigliucci V, O'Dowd G, Casey S, Egan D, Gibney S and Harkin A: Ketamine elicits sustained antidepressant-like activity via a serotonin-dependent mechanism. Psychopharmacology (Berl) 228: 157-166, 2013

88. Tan S, Lam WP, Wai MS, Yu WH and Yew DT: Chronic ketamine administration modulates midbrain dopamine system in mice. PLoS One 7: e43947, 2012.

89. Li N, Lee B, Liu RJ, Banasr M, Dwyer JM, Iwata M, Li XY, Aghajanian G and Duman RS: mTOR-dependent synapse formation underlies the rapid antidepressant effects of NMDA antagonists. Science 329: 959-964, 2010.

90. Loo C, Simpson B and MacPherson R: Augmentation strategies in electroconvulsive therapy. J ECT 26: 202-207, 2010.

91. Singh A and Kar SK: How electroconvulsive therapy works?: Understanding the neurobiological mechanisms. Clin Psychopharmacol Neurosci 15: 210-221, 2017.

92. Cano M, Cardoner N, Urretavizcaya M, Martínez-Zalacaín I, Goldberg X, Via E, Contreras-Rodríguez O, Camprodon J, de Arriba-Arnau A, Hernández-Ribas R, et al: Modulation of limbic and prefrontal connectivity by electroconvulsive therapy in treatment-resistant depression: A preliminary study. Brain Stimul 9: 65-71, 2016

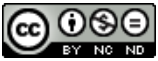

This work is licensed under a Creative Commons

Attribution-NonCommercial-NoDerivatives 4.0

International (CC BY-NC-ND 4.0) License. 